\title{
Topological States and Adiabatic Pumping in Quasicrystals
}

\author{
Yaacov E. Kraus, ${ }^{1}$ Yoav Lahini, ${ }^{2}$ Zohar Ringel, ${ }^{1}$ Mor Verbin, ${ }^{2}$ and Oded Zilberberg ${ }^{1}$ \\ ${ }^{1}$ Department of Condensed Matter Physics, Weizmann Institute of Science, Rehovot, 76100, Israel \\ ${ }^{2}$ Department of Physics of Complex Systems, Weizmann Institute of Science, Rehovot, 76100, Israel
}

(Received 29 March 2012; published 4 September 2012)

\begin{abstract}
The unrelated discoveries of quasicrystals and topological insulators have in turn challenged prevailing paradigms in condensed-matter physics. We find a surprising connection between quasicrystals and topological phases of matter: (i) quasicrystals exhibit nontrivial topological properties and (ii) these properties are attributed to dimensions higher than that of the quasicrystal. Specifically, we show, both theoretically and experimentally, that one-dimensional quasicrystals are assigned two-dimensional Chern numbers and, respectively, exhibit topologically protected boundary states equivalent to the edge states of a two-dimensional quantum Hall system. We harness the topological nature of these states to adiabatically pump light across the quasicrystal. We generalize our results to higher-dimensional systems and other topological indices. Hence, quasicrystals offer a new platform for the study of topological phases while their topology may better explain their surface properties.
\end{abstract}

DOI: 10.1103/PhysRevLett.109.106402

The discovery of topological insulators has sparked considerable interest in the study of topological phases of matter. Topological phases consist of various band insulators or superconductors that have gaps in their spectrum [1]. The hallmark of these novel phases is the emergence of topologically protected boundary phenomena, e.g., quantum pumping [2,3], surface states related to exotic models from particle physics [4], and quasiparticles with nonAbelian statistics [5]. Yet, realizations of these phases of matter are scarce [6-12].

Two systems belong to the same topological phase if they can be continuously deformed from one into the other without closing energy gaps. Consequently, at the interface between two topologically distinct systems, the energy gaps close by the appearance of localized boundary states. A classification of all the possible topological phases according to dimension and local symmetries was recently introduced [13]. For example, in the absence of any symmetries, all 1D systems belong to the topologically trivial phase, while in 2D there are the topological phases of the integer quantum Hall effect (IQHE) [14].

The order of quasicrystals (QCs) - nonperiodic structures with long-range order-can be seen as originating from periodic structures of a dimension higher than the physical one. For example, the 1D Fibonacci QC can be described as a projection of a 2D lattice on a line [15]. Remarkably, observed phenomena such as unconventional Bragg diffraction and the existence of phasons can be attributed to this higher dimension [15-17]. Remnants of the higher dimensionality appear as additional degrees of freedom (d.o.f.) in the form of shifts of the origin of the quasiperiodic order. These d.o.f. discern between QCs with the same quasiperiodic order, as they result in different patterns. However, they have no apparent influence on bulk properties and were therefore usually ignored.
PACS numbers: 71.23.Ft, 05.30.Rt, 42.70.Qs, $73.43 . \mathrm{Nq}$
In this Letter, we show that, due to the additional d.o.f., QCs exhibit nontrivial topological properties that are attributed to systems of a higher dimension. The topological properties of the QC manifest in two ways: (i) the existence of quantum phase transitions when continuously deforming between two topologically distinct QCs and (ii) the appearance of robust boundary states which traverse the bulk gaps as a function of the aforementioned shifts. Specifically, we demonstrate, both theoretically and experimentally, that 1D QCs exhibit topological properties that were, thus far, thought to be limited to 2D systems. Using photonic QCs, we observe localized boundary states, which manifest these topological properties. The topological nature of these boundary states is used to realize an adiabatic pumping of photons across the sample. Generalizations to various types of quasicrystals in 1D and higher dimensions are also discussed, suggesting the existence of topological effects on surfaces of $3 \mathrm{D}$ quasicrystals.

Let us begin with a specific QC, the 1D Aubry-André (AA) model [18] (also known as the Harper model). This is a 1D tight-binding model in which the on-site potential is modulated in space. It is described by the Hamiltonian

$$
H(\phi) \psi_{n}=t\left(\psi_{n+1}+\psi_{n-1}\right)+\lambda \cos (2 \pi b n+\phi) \psi_{n} .
$$

Here, $\psi_{n}$ is the wave function at site $n, t$ is the hopping amplitude, $\lambda$ is the modulation amplitude of the on-site potential, and $b$ controls the periodicity of the modulation. Whenever $b$ is irrational, the modulation is incommensurate with the lattice and the on-site term is quasiperiodic. Note that in this model the modulation phase $\phi$ embeds the d.o.f. mentioned above.

Figure 1 depicts a numerically calculated spectrum of the AA model as a function of $\phi$. Because of the incommensurate potential, the spectrum is broken into a fractal 


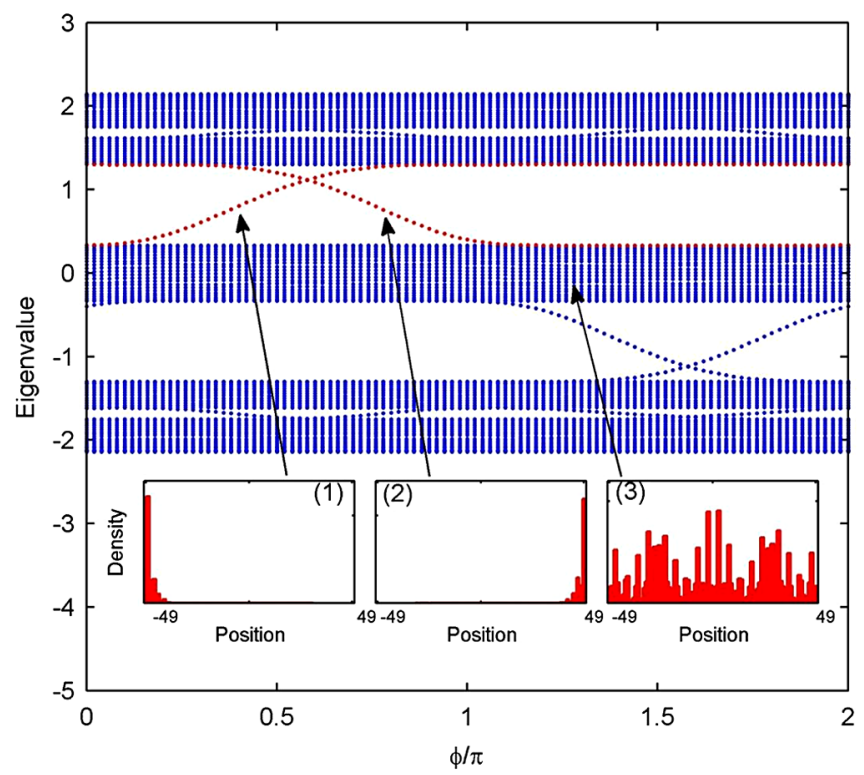

FIG. 1 (color online). The numerically calculated spectrum of Eq. (1) as a function of the phase $\phi$ for $t=1, \lambda=0.5$, $b=(\sqrt{5}+1) / 2$ (the golden mean), and $n=-49 \ldots 49$. The bulk of the spectrum remains fixed, whereas few modes, localized at the boundaries, sweep across the gaps. The insets depict the spatial density of typical eigenstates as a function of position along the 1D lattice: (1) a left boundary state, (2) a right boundary state, and (3) an extended state within the band.

set of bands and gaps [19]. Note that as a function of $\phi$ the bands are almost unchanged, but the gaps are crossed by a few modes. These states that reside within the gaps are boundary states, localized either on the left or on the right boundary of the system, as seen in insets (1) and (2), respectively. The states within the bands are typically extended, as depicted in inset (3). As we later show, these boundary states are the physical manifestation of the fact that the AA model belongs to a nontrivial topological phase.

We implemented the AA model in an optical setup using a quasiperiodic lattice of coupled single-mode waveguides. Because of a nonvanishing overlap between the evanescent modes, light that propagates along a waveguide can hop to its neighboring waveguides. In addition, along each waveguide a phase is accumulated in a rate determined by its refraction index. Hence, the propagation of light along the lattice is described by an equation which is identical to a tight-binding model, where the propagation axis, $z$, takes over the role of time, $i \partial_{z} \psi_{n}=H \psi_{n}$. Modulating the refraction index of the waveguides and the spacing between them controls the on-site and hopping terms of the Hamiltonian, respectively [20-22]. In particular, it enables a direct realization of the AA Hamiltonian of Eq. (1).

We produced an AA lattice with the parameters of Fig. 1 and $\phi=\pi / 2$ on a semiconductor (AlGaAs) substrate using standard photolithography methods [23]. We set the effective refraction index of each waveguide-by (a)

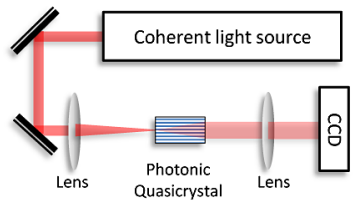

(b)

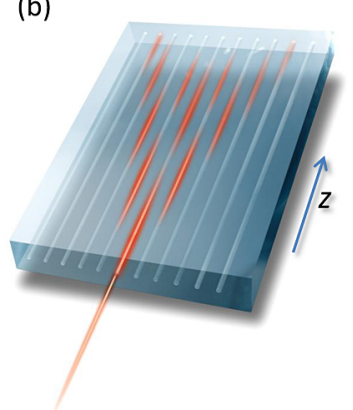

(d)
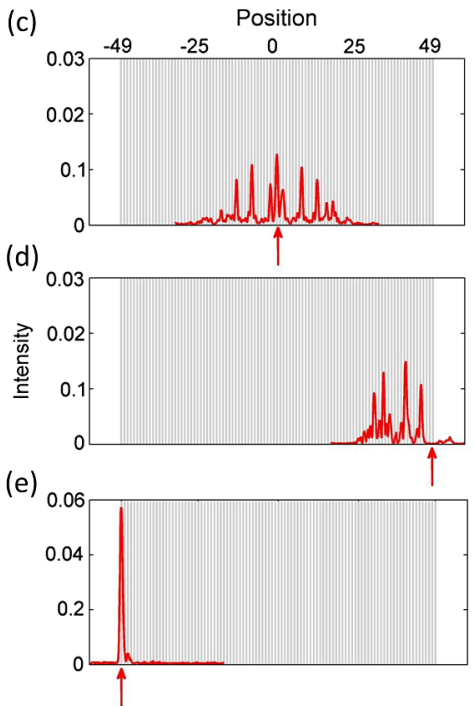

FIG. 2 (color online). Observation of topological boundary states in an Aubry-André photonic quasicrystal. (a) A sketch of the experimental setup. (b) An illustration of the conducted experiment. Light is injected into one of the waveguides and tunnels to neighboring waveguides as it propagates. (c)(e) Experimental observation of the left boundary state for $\phi=$ $\pi / 2$. Light was initially injected into a single waveguide (red arrows). The measured outgoing intensity is plotted versus the injection position along the lattice. (c),(d) An excitation at the middle of the lattice (site 0 ) and at the rightmost site (site 49) results in a significant spread. (e) For an excitation at the leftmost site (site -49), the light remains tightly localized at the boundary, marking the existence of a boundary state.

controlling its width - to fit the prescribed quasiperiodic pattern. We injected light into a single waveguide and measured the outgoing intensity distribution, as illustrated in Figs. 2(a) and 2(b). The experimental observations are depicted in Figs. 2(c)-2(e). Light injected into a lattice site in the middle of the lattice showed a significant expansion, due to the overlap of the injected wave function with the extended bulk eigenstates. Similarly, light injected into the rightmost lattice site showed considerable expansion. However, when the light was injected into the leftmost lattice site [see Fig. 2(e)], the intensity distribution remained tightly localized at the boundary, with the maximum intensity found at the leftmost waveguide, itself. This is a clear signature of the existence of a localized boundary state.

A consequence of the topological nature of this model is that all boundary states which reside within the same gap belong to the same mode. This can be seen by following the eigenenergy of some boundary state as a function of $\phi$. For example, take the right boundary state denoted in inset (2) of Fig. 1. Its mode is marked by red circles. This state remains localized on the right boundary as long as its energy remains within the gap. When the energy reaches the band, the state becomes extended. Notably, once the 
mode returns into the gap, it appears localized at the opposite boundary.

This property was used to realize adiabatic pumping of photons from one side of the lattice to the other. A convenient platform for this feat is the "off-diagonal" version of the AA model, which is described by the Hamiltonian

$$
\begin{aligned}
H_{\text {off }}(\phi) \psi_{n}= & t[1+\lambda \cos (2 \pi b n+\phi)] \psi_{n+1} \\
& +t[1+\lambda \cos (2 \pi b(n-1)+\phi)] \psi_{n-1} .
\end{aligned}
$$

While this model embeds its quasiperiodicity in the hopping term, it has topological characteristics similar to its previously discussed "diagonal" version [cf. Equation (1)]. The pumping takes place when $\phi$ is adiabatically swept along the propagation axis $z$.

In our implementation, we used waveguides written in bulk glass using femtosecond laser microfabrication technology [21]. The spacing between the waveguides was slowly modified along the propagation axis, thus realizing a sweep of $\phi$ in Eq. (2) [see Fig. 3(a)]. The length of the sample was $75 \mathrm{~mm}$, which is in our case 20 tunneling lengths, where the tunneling length is the characteristic scale for hopping, namely, $2 / t$ [23]. Figure 3(b) depicts the spectrum of the system as a function of $\phi$. In order to observe different stages of the pumping process, we fabricated a set of 50 samples for which the light was allowed to propagate shorter distances within the modulation. Correspondingly, in the $i$ th sample, $\phi$ is modulated from $0.35 \pi$ to $[0.35+1.4(i / 50)] \pi$. For each sample, light was injected to the rightmost site and the output intensity distribution was measured. The collected results are summarized in Fig. 3(c). The obtained intensity distributions are stacked incrementally according to their propagation distance, i.e., their final $\phi$. Thus, we reconstruct the light's trajectory along the full adiabatic process. It is evident that the injected light was pumped adiabatically across the QC from one boundary to the other [24].

We now turn to establish theoretically the topological properties of QCs. We start by showing that the observed boundary states are of topological origin by mapping the AA model to the lattice version of the 2D IQHE [19]. In the latter, electrons hop on a $2 \mathrm{D}$ rectangular lattice with nearest-neighbor hopping amplitudes $t$ and $t^{\prime}$ in the presence of a perpendicular magnetic field, with $b$ flux quanta threading each rectangle. Assuming one coordinate to be periodic and using the Landau gauge for the magnetic field, the system can be described by the Hamiltonian $\mathcal{H} \psi_{n, k}=$ $t\left(\psi_{n+1, k}+\psi_{n-1, k}\right)+2 t^{\prime} \cos (2 \pi b n+k a) \psi_{n, k}$, where $k$ is the momentum along the periodic coordinate with lattice spacing $a$ and $n$ is the location in real space along the second coordinate. The energy spectrum of $\mathcal{H}$ is gapped, and each gap is associated with a quantized Hall conductance $\sigma_{H}=\nu e^{2} / h$, with $\nu$ an integer [25] known as the Chern number [26]. The inclusion of disorder and distortions in the Hamiltonian does not alter $\sigma_{H}$, as long as the corresponding gap is maintained open [25-27]. Because of the fact that the energy gap must be closed in order for $\sigma_{H}$ to change its value, it can be used to classify different phases of the IQHE. Phases with different $\sigma_{H}$ are said to be topologically distinct. (a)

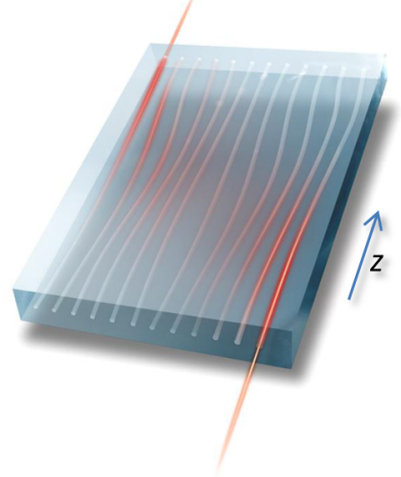

(b)

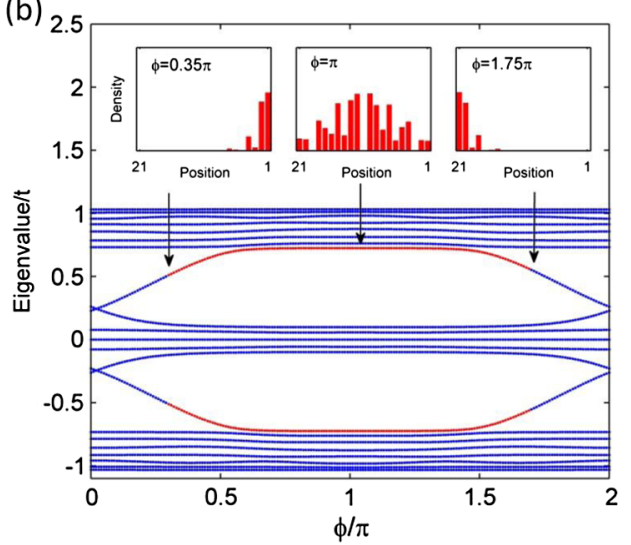

(c)

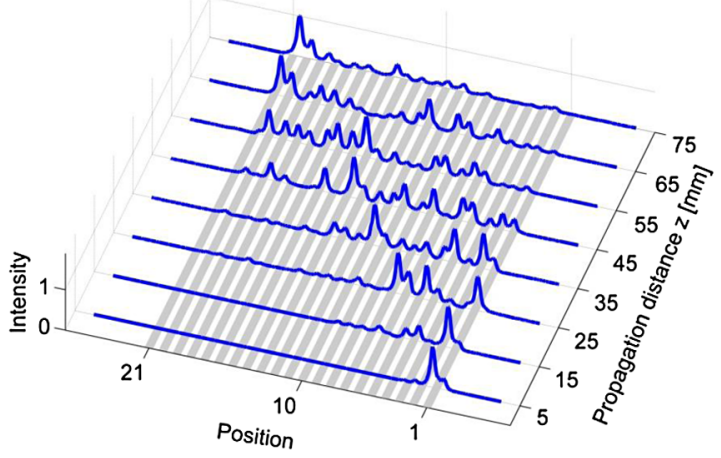

FIG. 3 (color online). Experimental observation of adiabatic pumping via topologically protected boundary states in a photonic quasicrystal. (a) An illustration of the adiabatically modulated photonic quasicrystal, constructed by slowly varying the spacing between the waveguides along the propagation axis $z$. Consequently, the injected light experiences an adiabatically modulated Hamiltonian, $H_{\text {off }}[\phi(z)]$, as it propagates and is pumped across the sample. (b) The spectrum of the model as a function of the phase $\phi$ for $t=40 / 75, \lambda=0.6, b=(\sqrt{5}+1) / 2$, and $n=1 \ldots 21$. In the experiment, $\phi$ was scanned between $0.35 \pi$ and $1.75 \pi$, marked by arrows (and red dots). The insets depict the spatial density of a boundary eigenstate as a function of the position at three different stages of the evolution: At $\phi=0.35 \pi$, the eigenstate is localized on the right boundary. At $\phi=\pi$, it is delocalized across the system, while at $\phi=1.75 \pi$ the state is again localized, but on the left boundary. (c) Experimental results: Light was injected into the rightmost waveguide (site 1$)$ at $z=0(\phi=0.35 \pi)$. The measured intensity distributions as a function of the position are presented at different stages of the adiabatic evolution, i.e., different propagation distances. It is evident that along the adiabatic evolution the light crossed the lattice from right to left. 
The physical manifestation of a nontrivial topological phase (i.e., $\sigma_{H} \neq 0$ ) is the emergence of robust chiral states along the edges of the sample. This general phenomenon is shared by many topological phases, not only the IQHE [28-30]. In the IQHE, on each edge, exactly $|\nu|$ edge states appear, and the energy of each edge state traverses the gap as $k$ varies from $-\pi / a$ to $\pi / a$. The signs of the group velocity of these edge states are opposite on opposite edges.

Turning back to the 1D AA model [cf. Equation (1)], we shall now observe that it inherits its robust boundary states from the 2D IQHE. For $\lambda=2 t^{\prime}$, the spectrum of $H(\phi)$ can be viewed as the $k$ th component of $\mathcal{H}$ at $k=\phi / a$. Therefore, by scanning $\phi$ from $-\pi$ to $\pi$, the spectrum of $H(\phi)$ reconstructs that of $\mathcal{H}$. Consequently, the chiral edge states that traverse the gaps as a function of $k$ appear now as 1D boundary states that traverse the gaps with $\phi$. Since these boundary states are of topological origin, the only way to eliminate them is to close the energy gap which they traverse. In particular, disorder which does not close an energy gap does not eliminate the corresponding boundary states.

Note that the topology guarantees the existence of boundary states only for intervals of $\phi$. Hence, it does not guarantee that for any QC pattern they indeed appear. This can be seen, for example, in Fig. 3(b), where for $\phi=0$ one finds states localized on both boundaries and for $\phi=\pi$ there are none. Additionally, changing the number of lattice sites or translating them (e.g., taking $n=5 \ldots 25$ instead of $n=1 \ldots 21$ ) may alter dramatically the intervals of $\phi$ for which boundary states appear.

So far, in order to witness the topological nature of the AA model, we had to scan $\phi$. Ostensibly, one can associate Chern numbers only to the union of all the $H(\phi)$ Hamiltonians. Indeed, per $H(\phi)$, a Chern density is assigned, while the Chern number involves integration of the Chern density over all $\phi$. However, we show that for QCs the Chern density is independent of $\phi$. Thus, the Chern number can be evaluated from the Chern density of any $H(\phi)$. Since the same quantized Chern number is associated with any $H(\phi)$, it topologically classifies it. This is somewhat analogous to the role of the Aharonov-Bohm flux in the IQHE $[23,25,30]$. The association of a Chern number with each QC is a key result of this work.

Proof of the above statement appears in the Supplemental Material [23]. Here, we show that the bulk spectrum is also independent of $\phi$. This simpler proof contains the essential ingredients of the former. Since $H(\phi)$ has a band structure, the spectrum is insensitive to lattice translations in the thermodynamic limit. From Eq. (1), it is evident that translating the lattice by $m$ sites is equivalent to shifting $\phi$ by $2 \pi(b m \bmod 1)$. Now the irrationality of $b$ comes into play. For a rational $b=p / q$, ( $b m$ mod1) has only $q$ different values for all possible translations. Thus, the band structure is guaranteed to be invariant only for these $q$ corresponding shifts of $\phi$. On the other hand, for irrational $b,(b m \bmod 1)$ samples the entire $[0,1]$ interval and the bands are invariant for any shift of $\phi$.

Thus, we arrive at the following conclusion: while in order to witness boundary effects the scanning over $\phi$ is required, the topological indices can be associated with any instance of a quasiperiodic pattern, i.e., any given $\phi$. These indices are, of course, the same for a given quasiperiodicity for all $\phi$ 's. Thus, the AA model is topologically classified. Consequently, two QCs with two different $b$ 's cannot be smoothly deformed from one to the other without closing the bulk gaps, since in the IQHE different $b$ 's result in different Chern numbers [23].

Until now, we focused on a specific model which we were able to map to the IQHE. However, our results could be easily generalized to any QC, such as the off-diagonal AA model and the Fibonacci QC. Moreover, our arguments apply to any dimension and any topological index without the need for establishing such a mapping [23]. Consider a $D$-dimensional QC with a tight-binding Hamiltonian with $d$ quasiperiodic terms, either hopping or on-site. These terms result in $d$ d.o.f. similar to the above $\phi$. In the context of topological properties, these d.o.f. could be treated as extra dimensions, yielding an overall effective dimension of $D+d$. Therefore, the Hamiltonian may belong to a nontrivial $D+d$-dimensional topological class.

We showed that quasicrystals exhibit new types of topological phases that were previously attributed only to systems of higher dimension. The study of these novel topological phases in 2D and 3D may lead to the discovery of new surface phenomena in atomic and photonic quasicrystals; e.g., 3D quasicrystalline materials may exhibit topological properties that would have appeared only in $6 \mathrm{D}$ periodic systems. Furthermore, our approach provides new tools for engineering photonic quasicrystals and especially for controlling their surface properties.

We thank Y. Silberberg, S. Huber, Y. Gefen, and E. Altman for useful discussions and N. Gontmakher for the illustration of the device. We especially thank Y. Silberberg for allowing us to conduct the experiments in his labs. We thank the U.S.-Israel Binational Science Foundation, the Minerva Foundation, Crown Photonics Center, ISF Grant No. 700822030182, and the IMOS Israel-Korea Grant for financial support. All authors contributed equally to this work.

[1] M.Z. Hasan and C. L. Kane, Rev. Mod. Phys. 82, 3045 (2010); X. L. Qi and S. C. Zhang, ibid. 83, 1057 (2011).

[2] R. B. Laughlin, Phys. Rev. B 23, 5632 (1981).

[3] B. A. Bernevig and S.C. Zhang, Phys. Rev. Lett. 96, 106802 (2006).

[4] X.-L. Qi, T. L. Hughes, and S.-C. Zhang, Phys. Rev. B 78, 195424 (2008).

[5] C. Nayak, S. H. Simon, A. Stern, M. Freedman, and S. Das Sarma, Rev. Mod. Phys. 80, 1083 (2008). 
[6] Y. Xia et al., Nature Phys. 5, 398 (2009).

[7] H. Zhang, C.-X. Liu, X.-L. Qi, X. Dai, Z. Fang, and S.-C. Zhang, Nature Phys. 5, 438 (2009).

[8] S. Chadov, X. Qi, J. Kübler, G. H. Fecher, C. Felser, and S. C. Zhang, Nature Mater. 9, 541 (2010).

[9] H. Lin, A. Wray, Y. Xia, S. Xu, S. Jia, R. J. Cava, A. Bansil, and M. Zahid Hasan, Nature Mater. 9, 546 (2010).

[10] M. Hafezi, E. A. Demler, M. D. Lukin, and J. M. Taylor, Nature Phys. 7, 907 (2011).

[11] T. Kitagawa, M. S. Rudner, E. Berg, and E. Demler, Phys. Rev. A 82, 033429 (2010).

[12] J. P. Dahlhaus, J. M. Edge, J. Tworzydło, and C. W. J. Beenakker, Phys. Rev. B 84, 115133 (2011).

[13] S. Ryu, A. P. Schnyder, A. Furusaki, and A. W. W. Ludwig, New J. Phys. 12, 065010 (2010).

[14] Y. Avron, R. Seiler, and B. Shapiro, Nucl. Phys. B265, 364 (1986).

[15] C. Janot, Quasicrystals (Clarendon, Oxford, 1994), 2nd ed.

[16] J. E. S. Socolar, T. C. Lubensky, and P. J. Steinhardt, Phys. Rev. B 34, 3345 (1986).

[17] B. Freedman, G. Bartal, M. Segev, R. Lifshitz, D. N. Christodoulides, and J.W. Fleischer, Nature (London) 440, 1166 (2006); O. Manela, M. Segev, D. N. Christodoulides, and D. Kip, New J. Phys. 12, 053017 (2010).

[18] S. Aubry and G. André, Ann. Isr. Phys. Soc. 3, 133 (1980).
[19] D. R. Hofstadter, Phys. Rev. B 14, 2239 (1976).

[20] D. N. Christodoulides, F. Lederer, and Y. Silberberg, Nature (London) 424, 817 (2003).

[21] A. Szameit, D. Blömer, J. Burghoff, T. Schreiber, T. Pertsch, S. Nolte, A. Tünnermann, and F. Lederer, Opt. Express 13, 10552 (2005).

[22] Y. Lahini, R. Pugatch, F. Pozzi, M. Sorel, R. Morandotti, N. Davidson, and Y. Silberberg, Phys. Rev. Lett. 103, 013901 (2009).

[23] See Supplemental Material at http://link.aps.org/ supplemental/10.1103/PhysRevLett.109.106402 for more details.

[24] This is analogous to Laughlin's pumping in the IQHE (see Ref. [2]). However, here a single mode is being pumped, while in the IQHE all the electrons of the occupied band contribute to the current and induce the charge pumping.

[25] D. J. Thouless, M. Kohmoto, M. P. Nightingale, and M. den Nijs, Phys. Rev. Lett. 49, 405 (1982).

[26] M. Kohmoto, Ann. Phys. (N.Y.) 160, 343 (1985).

[27] J. E. Avron, R. Seiler, and B. Simon, Phys. Rev. Lett. 51, 51 (1983).

[28] Y. Hatsugai, Phys. Rev. Lett. 71, 3697 (1993).

[29] C. L. Kane and E. J. Mele, Phys. Rev. Lett. 95, 146802 (2005).

[30] Z. Ringel and Y.E. Kraus, Phys. Rev. B 83, 245115 (2011). 